\title{
Influence of Spatially Variable Soil Permeability on Backward Erosion Piping
}

\author{
B.A. Robbins ${ }^{1}$, D.V. Griffiths ${ }^{2}$, and G.A. Fenton ${ }^{3}$ \\ ${ }^{1}$ U.S. Army Engineer Research and Development Center, Vicksburg, MS, U.S.A. \\ E-mail: bryant.a.robbins@usace.army.mil \\ ${ }^{2}$ Department of Civil and Environmental Engineering, Colorado School of Mines, Golden, CO, U.S.A. \\ E-mail: d.v.griffiths@mines.edu \\ ${ }^{3}$ Department of Engineering Mathematics, Dalhousie University, Halifax, NS, Canada. \\ E-mail: gordon.fenton@dal.ca
}

\begin{abstract}
Backward erosion piping (BEP) is a failure mechanism of serious concern for both dams and levees. Finite element models have been developed for analysis of BEP; however, these models often assume foundation soils are entirely homogenous. Recent laboratory investigations have demonstrated that spatial variability in soil substantially affects the BEP process, typically resulting in increased resistance to BEP. This paper presents a numerical investigation of the influence of spatial variation in permeability on BEP. A two-dimensional finite element model was developed for conducting plan view analyses of BEP in random permeability fields. Analyses results indicate that the probability of erosion progression is related to both the variance and correlation length of the permeability random field. BEP progression becomes less likely as the variance in permeability increases and the correlation length in the direction of flow decreases.
\end{abstract}

Keywords: Backward erosion piping; random fields; finite element.

\section{Introduction}

Backward erosion piping (BEP) refers to a type of internal erosion that occurs when shallow erosion pipes initiate at the downstream end of a water-retaining structure and gradually progress upstream towards the reservoir (Fig. 1a). The pipes progress further when the foundation sand is eroded by concentrated seepage forces near the upstream end of the pipes. If the erosion pipes reach the upstream reservoir, large increases in flow cause the pipes to quickly enlarge, ultimately leading to collapse and breach of the structure. As a significant number of dam failures have been caused by BEP (Foster et al. 2000; Lane 1935), BEP is often a failure mode of concern evaluated for existing dams and levees.

Recent research has led to the development of finite element (FE) models for simulation of BEP in uniform soils (e.g., Sellmeijer 2006; van Esch et al. 2013; Rotunno et al. 2017; Robbins and Griffiths 2018a). Meanwhile, laboratory experiments have demonstrated that BEP is highly sensitive to soil heterogeneities (Negrinelli et al. 2016) with BEP becoming less likely as soil variability increases. As such, models assuming homogenous soil profiles (1) may be overly conservative and (2) may not capture the process sufficiently to yield reliable probabilities of failure. Previous research aimed at assessing the influence of random soil properties on BEP has either not simulated the erosion process (Liu et al. 2017), simplified the erosion process (Kanning and Calle 2013), or was focused on other types of erosion (namely suffusion) that do not develop sequentially in the upstream direction (Liang et al. 2017). This paper presents an application of the Random Finite Element Method (RFEM), developed by Griffiths and Fenton (1993) and Fenton and Griffiths (1993), to the simulation of BEP in order to quantitatively assess how spatial variability in soil permeability influences pipe progression.

\section{Simulation Framework}

RFEM is a combination of finite element computations, random field generation, and Monte-Carlo analyses. The FE model properties are obtained from the random fields, and Monte-Carlo simulations are performed to generate distributions of results using many realizations of random fields. The FE model for BEP progression, the approach for random field generation, and the Monte-Carlo analyses are briefly described in the following sections.

\subsection{Two-dimensional simulation of BEP progression}

A FE program was developed for simulating the progression of erosion pipes in two-dimensional (2D) domains. Model formulations were developed for analysis in both plan and cross-section views (Robbins and Griffiths 2018 b). Only the modeling approach for plan view computations is discussed herein.

Consider the 2D plan view of the top of the sand layer shown in Fig. 1c. The domain represents the top of the sand layer immediately below the dam. The upstream edge at $x=0$ is at the upstream face of the dam. The

\author{
Proceedings of the 7th International Symposium on Geotechnical Safety and Risk (ISGSR) \\ Editors: Jianye Ching, Dian-Qing Li and Jie Zhang \\ Copyright (c) ISGSR 2019 Editors. All rights reserved. \\ Published by Research Publishing, Singapore.
}

ISBN: 978-981-11-2725-0; doi:10.3850/978-981-11-2725-0_bs2-cd 
downstream edge at $\mathrm{x}=10$ is located at the downstream toe of the dam. As a finite section of the foundation is being modeled, the other two boundaries (at $y=0$ and 10) are set as no flow boundaries.

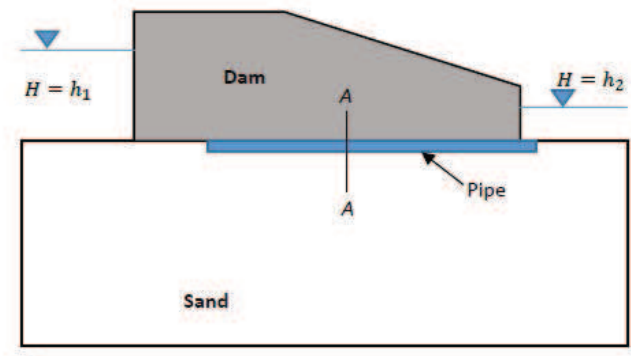

a.

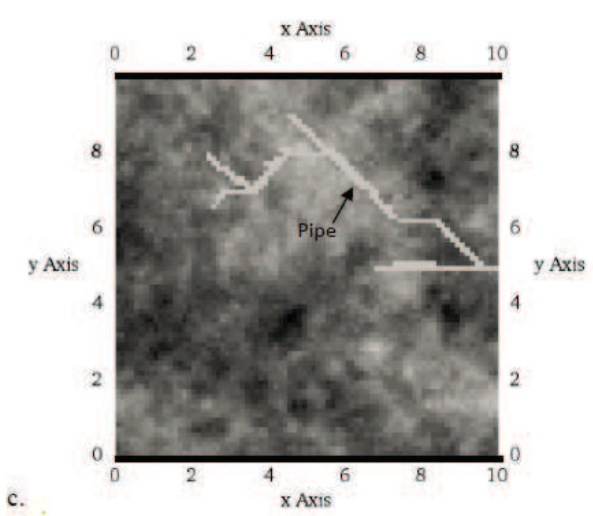

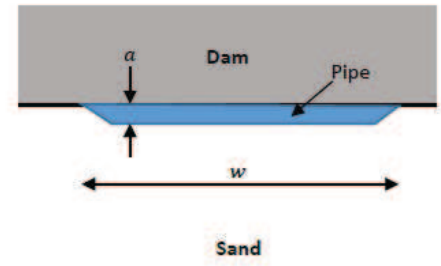

b.

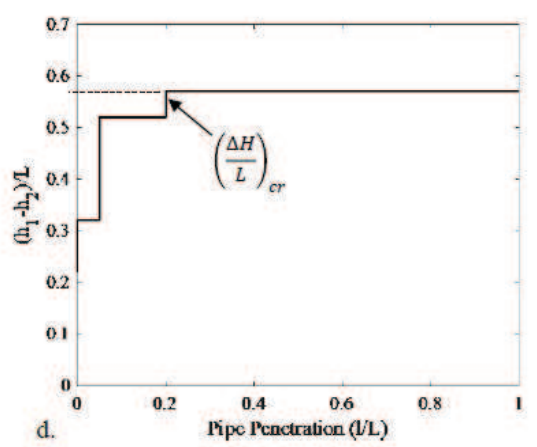

Figure 1. Illustration of (a) BEP passing beneath a dam, (b) Section A-A through the erosion pipe, (c) plan view of a pipe passing through the plane of sand with random permeability located immediately beneath the dam (dark represents low permeability), and (d) results of a single realization demonstrating selection of critical average gradient, defined as the global boundary head difference divided by the width of the mesh.

The progression of the pipe is simulated through a piecewise, steady state analysis of the groundwater flow and pipe flow as the pipe advances. The problem domain is separated into a soil domain of 4-node, quadrilateral soil elements and a pipe domain consisting of quadrilateral "pipe" elements with special treatment as described below. As the pipe progresses, soil elements are switched to pipe elements. In this manner, the pipe progression is simulated across a static mesh. The steady state groundwater flow is described by

$\frac{\partial}{\partial x}\left(k_{x}(x, y) \frac{\partial H(x, y)}{\partial x}\right)+\frac{\partial}{\partial y}\left(k_{y}(x, y) \frac{\partial H(x, y)}{\partial y}\right)=0$

where $k_{x}$ and $k_{y}$ designate the hydraulic conductivity in the $\mathrm{x}$ - and y-directions and $H$ is the hydraulic head (m) across the domain. As shown in Fig. 1b, the width-to-depth ratio $(w / a)$ of the erosion pipe is quite large (i.e., 840 according to van Beek 2015). Due to the pipe shape and typically shallow depths, it is commonly assumed that the flow in the pipe is viscous laminar flow between two parallel plates (van Esch et al. 2013; Sellmeijer 2006; Robbins and Griffiths 2018a). In this case, the pipe elements have an equivalent hydraulic conductivity given by

$k_{\text {pipe }}=\frac{a^{3} \rho_{w} g}{12 \mu}$

where $a, \rho_{w}, g$, and $\mu$ represent the pipe depth, density of water, gravitational acceleration, and dynamic viscosity of water, respectively. By assembling the pipe elements into the standard groundwater problem using Eq. 2 for the element permeability, the coupled groundwater flow and pipe flow equations are simultaneously solved.

However, in order to use Eq. 2, the depth of the erosion pipe must be known for each element. The depth of the pipe is determined by the critical shear stress, $\tau_{c}$, of the sand. If the hydraulic shear stress on the base of the pipe, $\tau_{b}$, is greater than the critical shear stress, the erosion pipe must deepen. The applied shear stress is given by 
$\tau_{b}=\frac{a \rho_{w} g i}{2}$

where $i$ designates the magnitude of the hydraulic gradient in the pipe. As the pipe depth in each element is not known a priori, it must be determined as part of the solution process. The pipe depth is initialized to a nominal depth of $3 d_{50}$, where $d_{50}$ denotes the median grain size of the sand, followed by Picard iterations over the depth $a$ until $\tau_{b} \leq \tau_{c}$ in all pipe elements. Once satisfied, a valid hydraulic solution is obtained for a given pipe geometry. To determine whether the pipe progresses further, the magnitude of the hydraulic gradient in every element $\left(i_{e l}\right)$ adjacent to the pipe is compared to the critical hydraulic gradient for pipe progression $\left(i_{c r}\right)$. If $i_{e l}>i_{c r}$ in an element, the element is switched to a pipe element; and the solution progresses to the next pipe progression step. The simulation steps for a complete analysis can be summarized as follows.

Simulation Algorithm Steps

1. Start at a low differential head to ensure $i_{c r}$ is not exceeded in many locations at once. This was found to be necessary to obtain realistic results. Initiate pipe by switching select elements to pipe elements. Two initiation approaches were studied: (1) initiation by a single element near the center of the downstream boundary ( $a t x=10, y=5$ ) and ( 2 ) initiation by all elements on the downstream boundary.

2. Solve groundwater and pipe flow using Eq. 1 and Eq. 2.

3. Calculate $\tau_{b}$ for pipe elements. If $\tau_{b}>\tau_{c}$ in any elements, increase $a$ in those elements and go to Step 2 .

4. Calculate $i_{e l}$ in all soil elements adjacent to the pipe. If $i_{e l}>i_{c r}$ in any elements, switch the element with the highest excess gradient $\left(i_{e l}-i_{c r}\right)$ to a pipe element. Go to Step 2.

5. If pipe has progressed through the domain, end simulation. Otherwise, increase the upstream head and go to Step 2.

By stepping through this algorithm, the percentage of pipe penetration through the domain can be determined for each applied head, as illustrated in Fig. 1d. The highest value of head obtained during the simulation is the critical head for BEP failure.

\subsection{Random field generation}

The primary spatial variables of interest are the soil permeability $(k)$ and the critical gradient for pipe progression $\left(i_{c r}\right)$. As both parameters are related to the grain size distribution of the soil (Schmertmann 2000), it is necessary to allow for the $k$-field and the $i_{c r}$-field to be cross-correlated. For uniformly graded soils, the fields are positively correlated, as both $k$ and $i_{c r}$ increase with grain size. For well graded soils, the fields can be positively or negatively correlated. Further, the $k$-field is a lognormally distributed variable due to the large variability in $k$ and the necessity for positive values while the $i_{c r}$-field is a normally distributed variable due to the moderate variability observed in lab measurements (Robbins et al. 2018).

To generate the cross-correlated random fields for each variable, the covariance matrix decomposition method was used (Fenton 1994; Fenton and Griffiths 2008). Two independent Gaussian random fields were generated using the Local Average Subdivision (LAS) method (Fenton and Vanmarke 1990) with specified correlation length $\theta=\theta_{x}=\theta_{y}$ and exponential covariance function. The covariance decomposition method was then used to generate a third Gaussian field that had a specified correlation to the first field. The two correlated fields were then transformed to the desired marginal distributions for each of the two variables of interest.

\subsection{Monte-Carlo Simulation}

In RFEM analysis, it is necessary to perform sufficient Monte-Carlo simulations to develop meaningful and reproducible statistics of output quantities of interest. For this particular problem, the average critical gradient at failure is of interest; therefore, distributions of the hydraulic gradients at failure were stored from each suite of simulations.

The values of all input variables considered are listed in Table 1. A Monte-Carlo analysis was conducted for each possible combination of input values. To determine the number of Monte-Carlo simulations to obtain stable output statistics, the mean and standard deviation of the critical average gradient were evaluated for the case with the highest permeability variance $\left(\sigma_{k} / \mu_{k}=3.0\right)$, since this case would require the largest number of simulations. Fig. 2 illustrates convergence for the two extreme correlation lengths. For the higher correlation length, it was observed that about 4,400 simulations were needed to get reasonable convergence of both the mean and the standard deviation, so this number was chosen for all the parametric studies that follow.

\section{Analysis Results}

In an attempt to isolate the influence of permeability on BEP progression, simulations were run with both constant values of $i_{c r}=0.30$ and random values of $i_{c r}$. Additionally, the results were found to be sensitive to whether or not the pipe initiation was restricted to a single location or allowed to start anywhere. The following sections present the results obtained for (i) random permeability only, (ii) random permeability with different initiation points, and (iii) combined influence of random permeability and random $i_{c r}$. 


\subsection{Influence of random permeability only}

Monte-Carlo simulations were first performed for all 25 possible combinations of input parameters (Table 1) using a deterministic value of $i_{c r}=0.3$ and the center downstream element for pipe initiation. The critical average gradient across the domain at BEP failure was recorded for each simulation. The cumulative distribution function (CDF) of the critical gradient was then plotted for each case to assess trends in the results. Select results are illustrated in Fig. 3. In general, the CDFs shifted to the right with increasing variance in the permeability random field, indicating that the probability of failure for a given gradient was decreasing with increasing variance. Additionally, by comparing Fig. 3a-d, it was also recognized that the CDFs shifted to the left with increasing correlation lengths. It appears that for a fixed coefficient of variation, the lowest probability of failure is obtained with the highest correlation length.

Table 1. Input values used in Monte-Carlo analysis of BEP progression.

\begin{tabular}{|r|l|r|l|}
\hline Domain Length $(L)$ and Width $(W)$ & $10 \mathrm{~m}$ & $\mu$ & $0.001 \mathrm{Ns} / \mathrm{m}^{2}$ \\
\hline Element Size & $0.125 \mathrm{~m}$ & $\mu_{i c r}$ & 0.3 \\
\hline$\mu_{k}$ & $1.0 \times 10^{-5} \mathrm{~m} / \mathrm{s}$ & $\sigma_{i c r}$ & 0.05 \\
\hline$d_{50}$ & $0.208 \mathrm{~mm}$ & $\theta / L$ & $0.05,0.1,0.25,0.5,1.0$ \\
\hline$\rho_{w}$ & $1,000 \mathrm{~kg} / \mathrm{m}^{3}$ & $\sigma_{k} / \mu_{k}$ & $0.25,0.5,1.0,2.0,3.0$ \\
\hline$\tau_{c}$ & $0.33 \mathrm{~Pa}$ & & \\
\hline
\end{tabular}
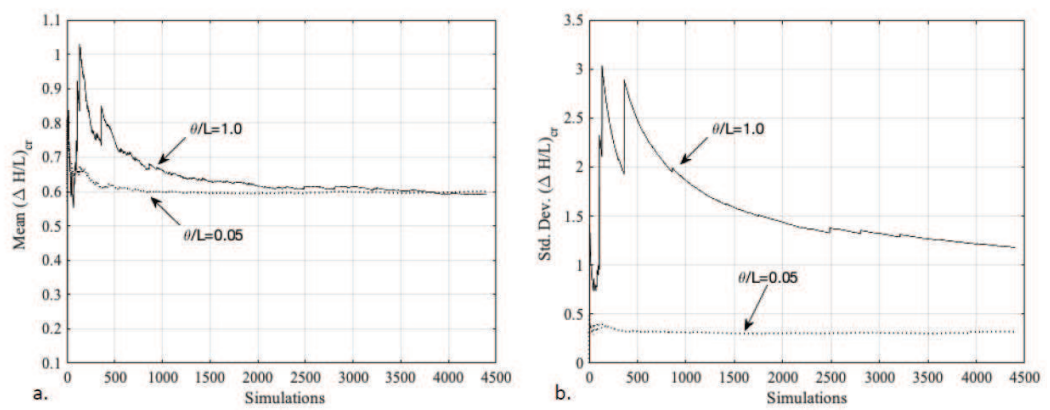

Figure 2. Convergence of (a) mean and (b) standard deviation of critical average gradient for BEP progression through random permeability field with $\sigma_{k} / \mu_{k}=3.0$ and deterministic $i_{c r}$ field of 0.30 .

\subsection{Influence of initiation condition}

For all previous analyses, the BEP pipe was initiated in a single element at the center of the downstream boundary. Alternatively, the pipe could be initiated anywhere along the entire downstream boundary. In this manner, the weakest location could be exploited. Simulations were run again for all 25 cases with the pipe initiated along the entire downstream boundary. As shown in Fig. 4a, allowing the pipe to start anywhere along the boundary increases the probability of failure for a given gradient. To quantify this shift for all 25 cases, the percent change in the median value of the critical average gradient was calculated for all 25 cases (Fig. 4b). Interestingly, the values decrease for all cases except for the case with the smallest variance. It is not immediately clear why initiation along the entire boundary caused an increase for the smallest variance, which is a topic of ongoing study. Regardless, the results demonstrate that the initiation condition can have a substantial influence on the calculated probabilities.

\subsection{Influence of random $i_{c r}$ on the results}

While random permeability has a significant influence on the piping calculations, permeability is not the only important variable. It is also of interest to see how random values of $i_{c r}$ influence the results (both correlated and uncorrelated). The 25 cases were rerun with random fields for $i_{c r}$ that were cross correlated to the permeability random field with correlation coefficients of -1.0, 0.0, and 1.0. Sample results are illustrated in Fig. 5. In general, for high permeability variance, the uncorrelated results were identical to the cases without random $i_{c r}$. For low permeability variance, the random $i_{c r}$ field had a significant influence. For all cases, positive correlation tended to shift the CDF to the right, and negative correlation shifted the CDF to the left.

\section{Conclusions}


Backward erosion piping is a failure mode for dams and levees that is substantially influenced by spatial variability in soil profiles. This study explored the influence of spatially variable permeability on the piping process through RFEM simulations. The results indicate that the probability of failure due to piping decreases as the variance in permeability increases and the correlation length decreases. Additionally, the calculated probability of failure was found to be higher when the pipe was allowed to initiate over a large area rather than at a single point. This is likely due to the large initiation zone ensuring that the weakest path is encountered. Lastly, inclusion of a random local gradient for pipe progression was also found to affect the results. However, the influence of the local critical gradient was not nearly as significant as the influence of the random permeability for the cases assessed. Future studies will continue to explore the relative significance of these primary variables.
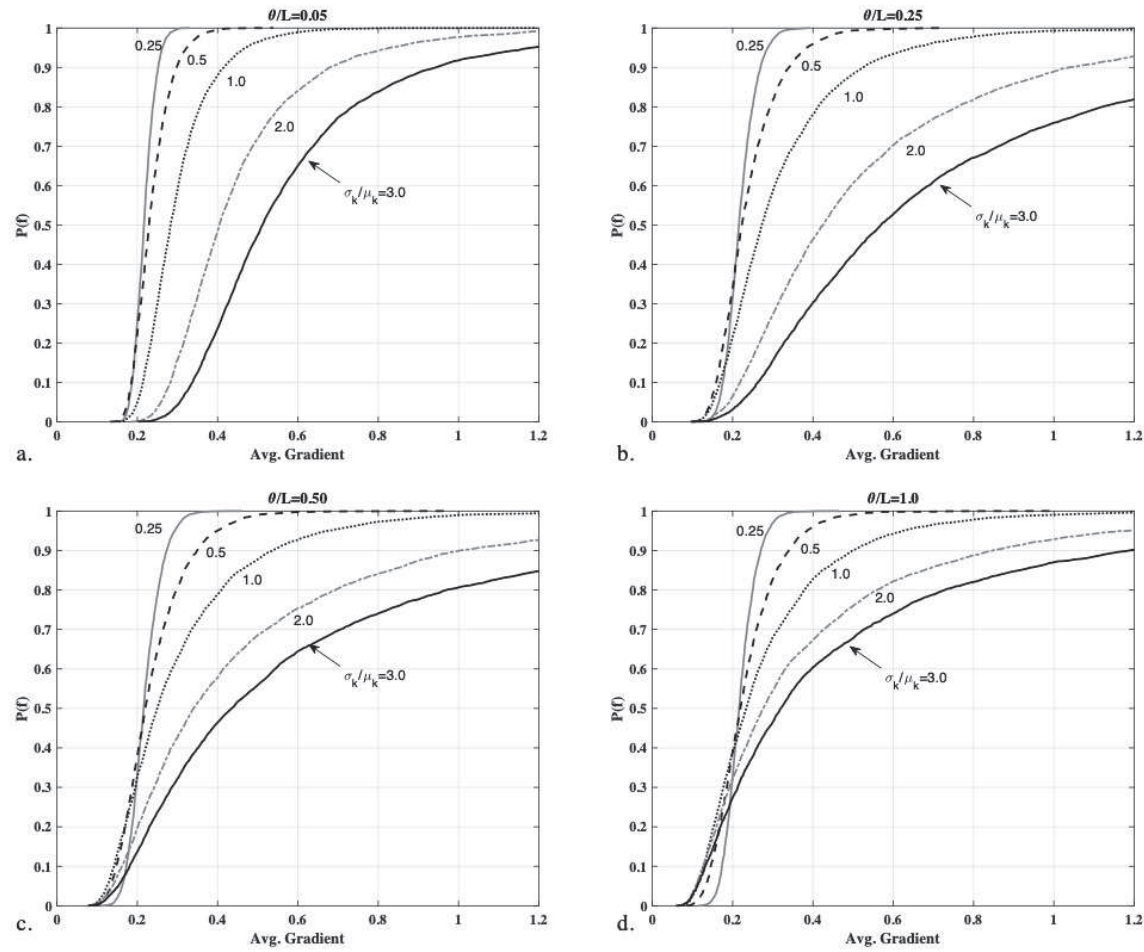

Figure 3. Distributions of critical average gradient with permeability as only random variable for (a) $\theta / L=0.05$, (b) $\theta / L=$ 0.25 , (c) $\theta / L=0.50$, and (d) $\theta / L=1.0$.

\section{References}

Fenton, G.A. (1994). Error evaluation of three random field generators. ASCE Journal of Engineering Mechanics, 120(12), 2478-2497.

Fenton, G.A. and Griffiths, D.V. (1993). Statistics of block conductivity through a simple bounded stochastic medium. Water Resources Research, 29(6), 1825-1830.

Fenton, G.A. and Griffiths, D.V. (2008). Risk Assessment in Geotechnical Engineering, John Wiley \& Sons.

Fenton, G.A. and Vanmarcke, E.H. (1990). Simulation of random fields via local average subdivision. ASCE Journal of Engineering Mechanics, 116(8), 1733-1749.

Foster, M., Fell, R., and Spannagle, M. (2000). The statistics of embankment dam failures and accidents. Canadian Geotechnical Journal, 37(5), 1000-1024.

Griffiths, D.V. and Fenton, G.A. (1993). Seepage beneath water retaining structures founded on spatially random soil. Géotechnique, 43(4), 577-587.

Kanning, W. and Calle, E.O.F. (2013). Derivation of a representative piping resistance parameter based on random field modelling of erosion paths. Georisk: Assessment and Management of Risk for Engineered Systems and Geohazards, 7(2), 99-109.

Lane, E.W. (1935). Security from under-seepage - masonry dams on earth foundations. Transactions of the American Society of Civil Engineers, 100(1), 1235-1272.

Liang, Y., Yeh, T.C.J., Wang, Y.L., Liu, M., Wang, J., and Hao, Y. (2017). Numerical simulation of backward erosion piping in heterogeneous fields. Water Resources Research, 53(4), 3246-3261. 
Liu, K., Vardon, P.J., and Hicks, M.A. (2017). Probabilistic analysis of seepage for internal stability of earth embankments. Environmental Geotechnics, (ahead of print), 1-13.

Negrinelli, G., van Beek, V.M., and Ranzi, R. (2016). Experimental and numerical investigation of backward erosion piping in heterogeneous sands. In Proceedings of ICSE7. Taylor and Francis, London, UK. 473-482.

Robbins, B.A. and Griffiths, D.V. (2018a). A simplified finite element implementation of the Sellmeijer model for backward erosion piping. In Proceedings of the $9^{\text {th }}$ European Conference on Numerical Methods in Geotechnical Engineering, Puerto, Portugal, June 25-27, 2018.

Robbins, B.A. and Griffiths, D.V. (2018b). Modelling of backward erosion piping in two-and three-dimensional domains. In European Working Group on Internal Erosion, Springer, Cham, 149-158.

Robbins, B.A., van Beek, V.M., López-Soto, J.F., Montalvo-Bartolomei, A.M., and Murphy, J. (2018). A novel laboratory test for backward erosion piping. International Journal of Physical Modelling in Geotechnics, 18(5), 266-279.

Rotunno, A.F., Callari, C., and Froiio, F. (2017). Computational modeling of backward erosion piping. Models, Simulation, and Experimental Issues in Structural Mechanics, Springer, 225-234.

Schmertmann, J.H. (2000). The no-filter factor of safety against piping through sands. In: Judgment and Innovation, edited by Francisco Silva and Edward Kavazanijian Jr. ASCE. 65-133.

Sellmeijer, J.B. (2006). Numerical computation of seepage erosion below dams (piping). In Proceedings of ICSE3 November 1-3, Amsterdam, The Netherlands.

van Beek, V.M. (2015). Backward Erosion Piping: Initiation and Progression. Dissertation. Delft University of Technology. van Esch, J.M., Sellmeijer, J.B., and Stolle, D. (2013). Modeling transient groundwater flow and piping under dikes and dams. 3rd International Symposium on Computational Geomechanics (ComGeo III), 9.
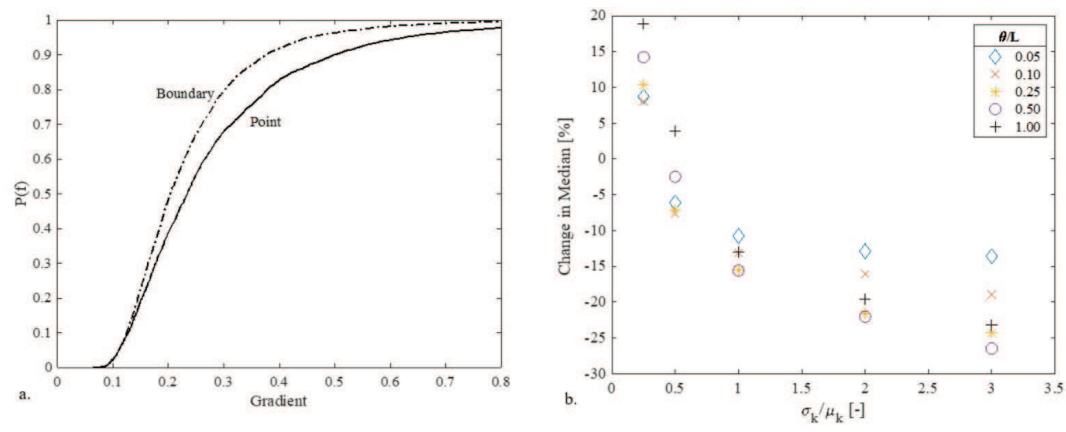

Figure 4. Influence of initiation condition on (a) CDF of critical gradient for $\sigma_{k} / \mu_{k}=1.0, \theta / L=1.0$ and (b) percent change in median critical gradient for all values of $\sigma_{k} / \mu_{k}$ and $\theta / L$.
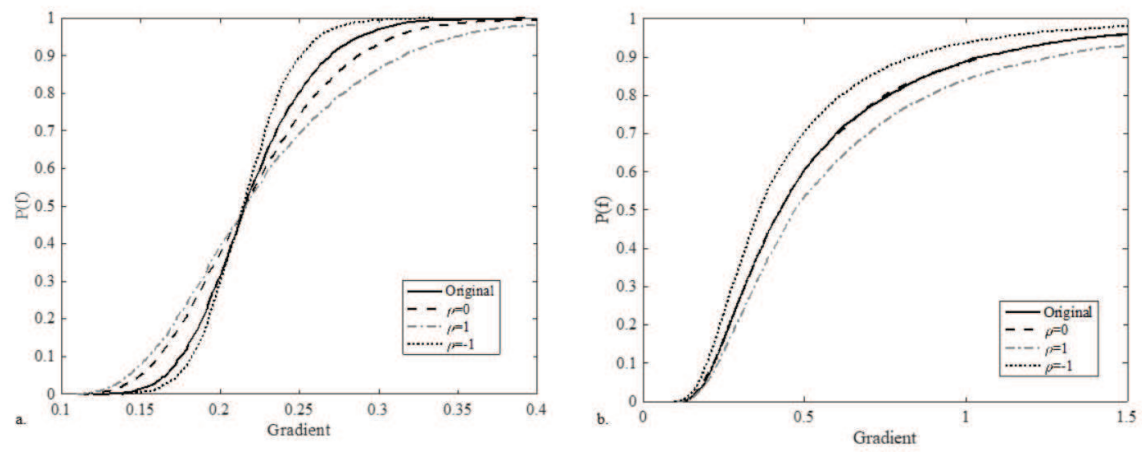

Figure 5. Combined influence of random $i_{c r}$ and $k$ fields on distribution of critical gradients for (a) $\sigma_{k} / \mu_{k}=0.25$ and $\theta / L=$ 0.25 and (b) $\sigma_{k} / \mu_{k}=2$ and $\theta / L=0.25$. 